\title{
The investigation of local weak solutions for a generalized Novikov equation
}

Shaoyong Lai*

\section{"Correspondence:}

laishaoy@swufe.edu.cn

Department of Mathematics,

Southwestern University of Finance

and Economics, Chengdu, 610074,

China

\begin{abstract}
The existence of local weak solutions for a generalized Novikov equation is established in the Sobolev space $H^{5}(R)$ with $1 \leq s \leq \frac{3}{2}$. The pseudo-parabolic regularization technique and several estimates derived from the equation itself are used to prove the existence.
\end{abstract}

MSC: 35Q35; 35Q51

Keywords: a generalized Novikov equation; local weak solution; existence

\section{Introduction}

Recently, many scholars have paid attention to the study of the integrable Novikov equation [1]

$$
v_{t}-v_{t x x}+4 v^{2} v_{x}=3 v v_{x} v_{x x}+v^{2} v_{x x x},
$$

which has a matrix Lax pair $[1,2]$ and is shown to be related to a negative flow in the Sawada-Kotera hierarchy. Several conservation quantities and a bi-Hamiltonian structure were found in [2]. Himonas and Holliman [3] applied the Galerkin-type approximation method to prove the well-posedness of strong solutions for Eq. (1) in the Sobolev space $H^{s}(R)$ with $s>\frac{3}{2}$ on both the line and the circle. Its Hölder continuity properties were studied in Himonas and Holmes [4]. The abstract Kato theorem was employed in Ni and Zhou [5] to show the existence and uniqueness of local strong solutions in the Sobolev space $H^{s}(R)$ with $s>\frac{3}{2}$. The persistence properties of the strong solution were found. The local well-posedness for the periodic Cauchy problem of the Novikov equation in the Sobolev space $H^{s}(R)$ with $s>\frac{5}{2}$ is done in Tiglay [6]. If the initial data are analytic, the existence and uniqueness of analytic solutions for Eq. (1) are obtained in [6]. It is worthy to mention that if the Sobolev index $s \geq 3$ and sign conditions hold, the orbit invariants are applied to show the existence of periodic global strong solution. The scattering theory is used by Hone $e t$ al. [7] to search for non-smooth explicit soliton solutions with multiple peaks for Eq. (1). This multiple peak property is common with the Camassa-Holm and Degasperis-Procesi equations (see [8-13]).

In this work, we study the following generalized dissipative Novikov equation:

$$
v_{t}-v_{t x x}+4 v^{2} v_{x}=3 v v_{x} v_{x x}+v^{2} v_{x x x}-\alpha v^{2 n+1}+\beta \partial_{x}\left(v_{x}^{2 N-1}\right),
$$

where $n \geq 0$ and $N \geq 1$ are nature numbers, constants $\alpha \geq 0$ and $\beta \geq 0$. The expression $\beta \partial_{x}\left(v_{x}^{2 N-1}\right)$ is a nonlinearly dissipative term. If $\alpha=\beta=0$, Eq. (2) becomes Eq. (1).

@2014 Lai; licensee Springer. This is an Open Access article distributed under the terms of the Creative Commons Attribution License (http://creativecommons.org/licenses/by/2.0), which permits unrestricted use, distribution, and reproduction in any medium, provided the original work is properly cited. 
Here, we address that all the generalized versions of the Novikov equation in previous works do not involve the nonlinearly dissipative terms $\partial_{x}\left(v_{x}^{2 N-1}\right)$. This is the motivation of our work to investigate Eq. (2). We establish the existence of local weak solutions for Eq. (2) in the lower order Sobolev space $H^{s}(R)$ with $1 \leq s \leq \frac{3}{2}$. Several estimates of solutions for the associated regularized equation for Eq. (2) are derived to prove the existence.

This paper is organized as follows. The main results are given in Section 2. In Section 3, we prove the local existence and uniqueness of solutions for the associated regularized Novikov equation (2) by using a contraction argument. In Section 4, we derive that Eq. (2) subject to the initial value $v_{0}(x)$ has a weak solution in the sense of a distribution.

\section{Main result}

The space of all infinitely differentiable functions $\phi(t, x)$ with compact support in $[0,+\infty) \times$ $R$ is denoted by $C_{0}^{\infty}$. We let $L^{p}=L^{p}(R)(1 \leq p<+\infty)$ be the space of all measurable functions $f(t, x)$ such that $\|f\|_{L^{p}}^{p}=\|f(t, \cdot)\|_{L^{p}}^{p}=\int_{R}|f(t, x)|^{p} d x<\infty$. We define $L^{\infty}=L^{\infty}(R)$ with the standard norm $\|f\|_{L^{\infty}}=\|f(t, \cdot)\|_{L^{\infty}}=\inf _{m(e)=0} \sup _{x \in R \backslash e}|f(t, x)|$. For any real number $s$, we let $H^{s}=H^{s}(R)$ denote the Sobolev space with the norm defined by

$$
\|f\|_{H^{s}}=\|f(t, \cdot)\|_{H^{s}}=\left(\int_{R}\left(1+|\xi|^{2}\right)^{s}|\hat{f}(t, \xi)|^{2} d \xi\right)^{\frac{1}{2}}<\infty
$$

where $\hat{f}(t, \xi)=\int_{R} e^{-i x \xi} f(t, x) d x$. Let $C\left([0, T] ; H^{s}(R)\right)$ denote the class of continuous functions from $[0, T]$ to $H^{s}(R)$ where $T>0$. We set $\Lambda=\left(1-\partial_{x}^{2}\right)^{\frac{1}{2}}$. For simplicity, throughout this article, we let $c$ denote any positive constant which is independent of parameter $\varepsilon$.

For the generalized Novikov equation (2), we consider the Cauchy problem of its associated regularized equation

$$
\left\{\begin{aligned}
v_{t}-v_{t x x}+\varepsilon v_{t x x x x}= & -4 v^{2} v_{x}+3 v v_{x} v_{x x}+v^{2} v_{x x x}-\alpha v^{2 n+1}+\beta \partial_{x}\left(v_{x}^{2 N-1}\right) \\
= & -\frac{4}{3} \partial_{x}\left(v^{3}\right)+\frac{1}{3} \partial_{x}^{3}\left(v^{3}\right)-2 \partial_{x}\left(v v_{x}^{2}\right) \\
& +v v_{x} v_{x x}-\alpha v^{2 n+1}+\beta \partial_{x}\left(v_{x}^{2 N-1}\right) \\
v(0, x)=v_{0}(x) . &
\end{aligned}\right.
$$

Before giving the main result of this work, we give two lemmas which are related to the regularized problem (3).

Lemma 2.1 Assume $v_{0}(x) \in H^{s}(R)$ with $s>\frac{3}{2}$. Then there exists a unique solution to Cauchy problem (3) in the space $C\left([0, T] ; H^{s}(R)\right)$ where $T>0$ depends on $\left\|\nu_{0}\right\|_{H^{s}(R)}$. If $s \geq 2$, the solution $v \in C\left([0,+\infty) ; H^{s}(R)\right)$.

For a real number $s$ with $s>0$, suppose that the function $v_{0}(x) \in H^{s}(R)$, and let $v_{\varepsilon 0}$ be the convolution $v_{\varepsilon 0}=\varphi_{\varepsilon} \star v_{0}$ of the function $\varphi_{\varepsilon}(x)=\varepsilon^{-\frac{1}{4}} \varphi\left(\varepsilon^{-\frac{1}{4}} x\right)$ and $v_{0}$ such that the Fourier transform $\widehat{\varphi}$ of $\varphi$ satisfies $\widehat{\varphi} \in C_{0}^{\infty}, \widehat{\varphi(\xi)} \geq 0$, and $\widehat{\varphi(\xi)}=1$ for any $\xi \in(-1,1)$. Then we have $v_{\varepsilon 0}(x) \in C^{\infty}$. It follows from Lemma 2.1 that for each $\varepsilon$ satisfying $0<\varepsilon<\frac{1}{4}$, the Cauchy problem

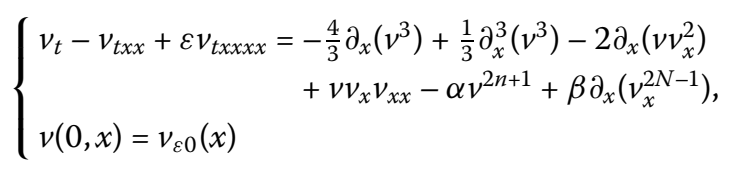

has a unique solution $v_{\varepsilon}(t, x) \in C^{\infty}\left([0, \infty) ; H^{\infty}(R)\right)$. 
Lemma 2.2 If $v_{0}(x) \in H^{s}(R)$ with $s \in\left[1, \frac{3}{2}\right]$ such that $\left\|v_{0 x}\right\|_{L^{\infty}}<\infty$. Let $v_{\varepsilon 0}=\varphi_{\varepsilon} \star v_{0}$. Then there exist two constants $T>0$ and c independent of $\varepsilon$ such that the solution $v_{\varepsilon}$ of problem (4) satisfies $\left\|v_{\varepsilon x}\right\|_{L^{\infty}} \leq c$ for any $t \in[0, T)$.

We write the Cauchy problem for Eq. (2)

$$
\left\{\begin{aligned}
v_{t}-v_{t x x}= & -\frac{4}{3} \partial_{x}\left(v^{3}\right)+\frac{1}{3} \partial_{x}^{3}\left(v^{3}\right)-2 \partial_{x}\left(v v_{x}^{2}\right) \\
& +v v_{x} v_{x x}-\alpha v^{2 n+1}+\beta \partial_{x}\left(v_{x}^{2 N-1}\right), \\
v(0, x)= & v_{0}(x), \quad x \in R
\end{aligned}\right.
$$

Now we state the main result of this work.

Theorem 2.1 Assume $v_{0}(x) \in H^{s}$ with $1 \leq s \leq \frac{3}{2}$ and $\left\|v_{0 x}\right\|_{L^{\infty}}<\infty$. Then there exists a $T>0$ such that problem (5) has at least one weak solution $v(t, x) \in L^{2}\left([0, T], H^{s}(R)\right)$ in the sense of distribution and $v_{x} \in L^{\infty}([0, T] \times R)$.

\section{Proof of Lemma 2.1}

Lemma 3.1 Let $r$ and $q$ be real numbers such that $-r<q \leq r$, then

$$
\begin{aligned}
& \left\|v_{1} v_{2}\right\|_{H^{q}} \leq c\left\|v_{1}\right\|_{H^{r}}\left\|v_{2}\right\|_{H^{q}}, \quad \text { if } r>\frac{1}{2}, \\
& \left\|v_{1} v_{2}\right\|_{H^{r+q-\frac{1}{2}}} \leq c\left\|v_{1}\right\|_{H^{r}}\left\|v_{2}\right\|_{H^{q}}, \quad \text { if } r<\frac{1}{2} .
\end{aligned}
$$

This lemma can be found in [11] or [12].

Proof of Lemma 2.1 Defining the operator $D=\left(1-\partial_{x}^{2}+\varepsilon \partial_{x}^{4}\right)^{-1}$, we know that $D: H^{s} \rightarrow H^{s+4}$ is a bounded linear operator. Using the operator $D$ to both sides of the first equation of problem (3) and then integrating the resultant equation over the interval $(0, t)$ give rise to

$$
\begin{aligned}
v(t, x)= & v_{0}(x)+\int_{0}^{t} D\left[\partial_{x}\left(-\frac{4}{3} v^{3}-2 v v_{x}^{2}\right)+\frac{1}{3} \partial_{x}^{3}\left(v^{3}\right)\right. \\
& \left.+v v_{x} v_{x x}-\alpha v^{2 n+1}+\beta \partial_{x}\left(v_{x}^{2 N-1}\right)\right] d t .
\end{aligned}
$$

We choose that $v$ and $g$ belong to the closed ball $B_{R_{0}}(0)$ of radius $R_{0}$ about the zero function in $C\left([0, T] ; H^{s}(R)\right)$ and $\Gamma$ is the operator on the right-hand side of Eq. (6). For fixed $t \in$ $[0, T]$, we obtain

$$
\begin{aligned}
& \| \int_{0}^{t} D\left[\partial_{x}\left(-\frac{4}{3} v^{3}-2 v v_{x}^{2}\right)+\frac{1}{3} \partial_{x}^{3}\left(v^{3}\right)+v v_{x} v_{x x}-\alpha v^{2 n+1}+\beta \partial_{x}\left(v_{x}^{2 N-1}\right)\right] d t \\
& \quad-\int_{0}^{t} D\left[\partial_{x}\left(-\frac{4}{3} g^{3}-2 g g_{x}^{2}\right)+\frac{1}{3} \partial_{x}^{3}\left(g^{3}\right)+g g_{x} g_{x x}-\alpha g^{2 n+1}+\beta \partial_{x}\left(g_{x}^{2 N-1}\right)\right] d t \|_{H^{s}} \\
& \leq C_{1} T\left(\sup _{0 \leq t \leq T}\left\|v^{3}-g^{3}\right\|_{H^{s}}+\sup _{0 \leq t \leq T}\left\|v^{2 n+1}-g^{2 n+1}\right\|_{H^{s}}\right. \\
& \quad+\sup _{0 \leq t \leq T}\left\|D \partial_{x}\left(v v_{x}^{2}-g g_{x}^{2}\right)\right\|_{H^{s}}+\sup _{0 \leq t \leq T}\left\|D\left(v v_{x} v_{x x}-g g_{x} g_{x x}\right)\right\|_{H^{s}} \\
& \left.\quad+\sup _{0 \leq t \leq T}\left\|D \partial_{x}\left(v_{x}^{2 N-1}-g_{x}^{2 N-1}\right)\right\|_{H^{s}}\right),
\end{aligned}
$$


where $C_{1}$ may depend on $\varepsilon$. Applying the algebraic property of the space $H^{s}(R)$ with $s>\frac{1}{2}$, we will give estimates for the right-hand side of inequality (7). In fact, we have

$$
\begin{aligned}
&\left\|v^{2 n+1}-g^{2 n+1}\right\|_{H^{s}} \leq c \| v-g\left\|_{H^{s}} \sum_{j=0}^{2 n}\right\| v\left\|_{H^{s}}^{j}\right\| g \|_{H^{s}}^{2 n-j} \\
& \leq C R_{0}^{2 n}\|v-g\|_{H^{s}}, \\
&\left\|D \partial_{x}\left(v_{x}^{2 N-1}-g_{x}^{2 N-1}\right)\right\|_{H^{s}}\left\|D \partial_{x}\left[\left(v_{x}-g_{x}\right) \sum_{j=1}^{2 N-2} v_{x}^{j} g_{x}^{2 N-2-j}\right]\right\|_{H^{s}} \\
& \leq\left\|\left(v_{x}-g_{x}\right) \sum_{j=1}^{2 N-2} v_{x}^{j} g_{x}^{2 N-2-j}\right\|_{H^{s-1}} \\
& \leq\left\|v_{x}-g_{x}\right\|_{H^{s-1}}\left\|\sum_{j=1}^{2 N-2} v_{x}^{j} g_{x}^{2 N-2-j}\right\|_{H^{s-1}} \\
& \leq C R_{0}^{2 N-2}\|v-g\|_{H^{s}(R)}
\end{aligned}
$$

and

$$
\begin{aligned}
\left\|D \partial_{x}\left[v v_{x}^{2}-g g_{x}^{2}\right]\right\|_{H^{s}} & \leq\left\|D \partial_{x}\left[v\left(v_{x}^{2}-g_{x}^{2}\right)\right]\right\|_{H^{s}}+\left\|D \partial_{x}\left[g_{x}^{2}(v-g)\right]\right\|_{H^{s}} \\
& \leq C\left(\left\|v\left(v_{x}^{2}-g_{x}^{2}\right)\right\|_{H^{s-1}}+\left\|g_{x}^{2}(v-g)\right\|_{H^{s-1}}\right) \\
& \leq C R_{0}^{2}\|v-g\|_{H^{s}}
\end{aligned}
$$

The first inequality of Lemma 3.1 yields

$$
\begin{aligned}
\left\|D\left(v v_{x} v_{x x}-g g_{x} g_{x x}\right)\right\|_{H^{s}} & =\left\|\frac{1}{2} D\left[v\left(v_{x}^{2}\right)_{x}-g\left(g_{x}^{2}\right)_{x}\right]\right\|_{H^{s}} \\
& \leq \frac{1}{2}\left(\left\|D\left[v\left(v_{x}^{2}-g_{x}^{2}\right)_{x}\right]\right\|_{H^{s}}+\left\|D\left[\left(g_{x}^{2}\right)_{x}(v-g)\right]\right\|_{H^{s}}\right) \\
& \leq C\left(\left\|v\left(v_{x}^{2}-g_{x}^{2}\right)_{x}\right\|_{H^{s-2}}+\left\|\left(g_{x}^{2}\right)_{x}(v-g)\right\|_{H^{s-2}}\right) \\
& \leq C\left(\|v\|_{H^{s}}\left\|v_{x}^{2}-g_{x}^{2}\right\|_{H^{s-1}}+\left\|g_{x}^{2}\right\|_{H^{s-1}}\|v-g\|_{H^{s}}\right) \\
& \leq C R_{0}^{2}\|v-g\|_{H^{s}} .
\end{aligned}
$$

From Eqs. (7)-(11), we have

$$
\|\Gamma v-\Gamma g\|_{H^{s}} \leq T C_{2} \max \left(C R_{0}^{2}, C R_{0}^{2 n}, C R_{0}^{2 N-2}\right)\|v-g\|_{H^{s}},
$$

where $C_{2}$ is independent of $0<t<T$. Choosing $T$ sufficiently small such that $T C_{2} \max \left(C R_{0}^{2}\right.$, $\left.C R_{0}^{2 n}, C R_{0}^{2 N-2}\right)<1$, we know that $\Gamma$ is a contracted mapping and $\|\Gamma v\|_{H^{s}} \leq T C_{2} \max \left(C R_{0}^{2}\right.$, $\left.C R_{0}^{2 n}, C R_{0}^{2 N-2}\right)\|v\|_{H^{s}}<R_{0}$. This means that $\Gamma$ maps $B_{R_{0}}(0)$ to itself. By the contractionmapping principle, we see that the mapping $\Gamma$ has a unique fixed point $v$ in $B_{R_{0}}(0)$.

For $s \geq 2$, using the first equation of system (3) gives rise to

$$
\frac{d}{d t} \int_{R}\left(v^{2}+v_{x}^{2}+\varepsilon v_{x x}^{2}+2 \int_{0}^{t}\left[\alpha v^{2 n+2}+\beta v_{x}^{2 N}\right] d \tau\right) d x=0,
$$


which derives the conservation law

$$
\begin{aligned}
& \int_{R}\left(v^{2}+v_{x}^{2}+\varepsilon v_{x x}^{2}+2 \int_{0}^{t}\left[\alpha v^{2 n+2}+\beta v_{x}^{2 N}\right] d \tau\right) d x \\
& =\int_{R}\left(v_{0}^{2}+v_{0 x}^{2}+\varepsilon v_{0 x x}^{2}\right) d x .
\end{aligned}
$$

The global existence result follows a routine argument by using the integral (14).

\section{Proofs of Lemma 2.2 and Theorem 2.1}

Lemma 4.1 (Kato and Ponce [14]) If $r \geq 0$, then $H^{r} \cap L^{\infty}$ is an algebra. Moreover,

$$
\|v g\|_{H^{r}} \leq c\left(\|v\|_{L^{\infty}}\|g\|_{H^{r}}+\|v\|_{H^{r}}\|g\|_{L^{\infty}}\right)
$$

where $c$ is a constant depending only on $r$.

Lemma 4.2 (Kato and Ponce [14]) Let $r>0$. If $v \in H^{r} \cap W^{1, \infty}$ and $g \in H^{r-1} \cap L^{\infty}$, then

$$
\left\|\left[\Lambda^{r}, v\right] g\right\|_{L^{2}} \leq c\left(\left\|\partial_{x} v\right\|_{L^{\infty}}\left\|\Lambda^{r-1} g\right\|_{L^{2}}+\left\|\Lambda^{r} v\right\|_{L^{2}}\|g\|_{L^{\infty}}\right) .
$$

Lemma 4.3 Let $s \geq 2$ and the function $v(t, x)$ is a solution of problem (3) and the initial data $v_{0}(x) \in H^{s}(R)$. Then

$$
\begin{aligned}
\|v\|_{H^{1}}^{2} & \leq c \int_{R}\left(v^{2}+v_{x}^{2}+\varepsilon v_{x x}^{2}+2 \int_{0}^{t}\left[\alpha v^{2 n+2}+\beta v_{x}^{2 N}\right] d \tau\right) d x \\
& =c \int_{R}\left(v_{0}^{2}+v_{0 x}^{2}+\varepsilon v_{0 x x}^{2}\right) d x .
\end{aligned}
$$

If $q \in[0, s-1]$, there is a constant $c$ independent of $\varepsilon$ such that

$$
\begin{aligned}
& \int_{R}\left[\left(\Lambda^{q+1} v\right)^{2}+\varepsilon\left(\Lambda^{q} v_{x x}\right)^{2}\right] d x \\
& \leq \int_{R}\left[\left(\Lambda^{q+1} v_{0}\right)^{2}+\varepsilon\left(\Lambda^{q} v_{0 x x}\right)^{2}\right] d x \\
& \quad+c \int_{0}^{t}\|v\|_{H^{q+1}}^{2}\left(\left\|v_{x}\right\|_{L^{\infty}}\|v\|_{L^{\infty}}+\left\|v_{x}\right\|_{L^{\infty}}^{2}+\|v\|_{L^{\infty}}^{2 n}+\left\|v_{x}\right\|_{L^{\infty}}^{2 N-2}\right) d \tau .
\end{aligned}
$$

If $q \in[0, s-1]$, there is a constant $c$ independent of $\varepsilon$ such that

$$
\begin{gathered}
(1-2 \varepsilon)\left\|v_{t}\right\|_{H^{q}} \leq c\|v\|_{H^{q+1}}\left(\|v\|_{L^{\infty}}\|v\|_{H^{1}}+\|v\|_{L^{\infty}}\left\|v_{x}\right\|_{L^{\infty}}\right. \\
\left.+\|v\|_{L^{\infty}}^{2 n}+\left\|v_{x}\right\|_{L^{\infty}}^{2}+\left\|v_{x}\right\|_{L^{\infty}}^{2 N-2}\right) .
\end{gathered}
$$

Proof By the identity $\|v\|_{H^{1}}^{2}=\int_{R}\left(v^{2}+v_{x}^{2}\right) d x$ and Eq. (14) one derives Eq. (15).

Using $\partial_{x}^{2}=-\Lambda^{2}+1$ and the Parseval equality, we have

$$
\int_{R} \Lambda^{q} v \Lambda^{q} \partial_{x}^{3}\left(v^{3}\right) d x=3 \int_{R}\left(\Lambda^{q} v\right) \Lambda^{q}\left(v^{2} v_{x}\right) d x-3 \int_{R}\left(\Lambda^{q+1} v\right) \Lambda^{q+1}\left(v^{2} v_{x}\right) d x
$$


If $q \in(0, s-1]$, using $\left(\Lambda^{q} v\right) \Lambda^{q}$ to multiply both sides of the first equation of system (3) and combining with Eq. (18), we get

$$
\begin{aligned}
\frac{1}{2} \frac{d}{d t} \int_{R}\left(\left(\Lambda^{q} v\right)^{2}+\left(\Lambda^{q} v_{x}\right)^{2}+\varepsilon\left(\Lambda^{q} v_{x x}\right)^{2}\right) d x \\
=-3 \int_{R}\left(\Lambda^{q} v\right) \Lambda^{q}\left(v^{2} v_{x}\right) d x-\int_{R}\left(\Lambda^{q+1} v\right) \Lambda^{q+1}\left(v^{2} v_{x}\right) d x \\
\quad+2 \int_{R}\left(\Lambda^{q} v_{x}\right) \Lambda^{q}\left(v v_{x}^{2}\right) d x+\int_{R}\left(\Lambda^{q} v\right) \Lambda^{q}\left(v v_{x} v_{x x}\right) d x \\
\quad-\alpha \int_{R} \Lambda^{q} v \Lambda^{q}\left(v^{2 n+1}\right) d x+\beta \int_{R} \Lambda^{q} v \Lambda^{q} \partial_{x}\left(v_{x}^{2 N-1}\right) d x
\end{aligned}
$$

We will estimate every term on the right-hand side of Eq. (19), separately. By using CauchySchwarz inequality and Lemmas 4.1 and 4.2 , we have

$$
\begin{aligned}
\left|\int_{R}\left(\Lambda^{q} v\right) \Lambda^{q}\left(v^{2} v_{x}\right) d x\right| & =\left|\int_{R} \Lambda^{q} v\left[\Lambda^{q}\left(v^{2} v_{x}\right)-v^{2} \Lambda^{q} v_{x}\right] d x+\int_{R}\left(\Lambda^{q} v\right) v^{2} \Lambda^{q} v_{x} d x\right| \\
& \leq c\|v\|_{H^{q}}\|v\|_{L^{\infty}}\left\|v_{x}\right\|_{L^{\infty}}\|v\|_{H^{q}}+\|v\|_{L^{\infty}}\left\|v_{x}\right\|_{L^{\infty}}\left\|\Lambda^{q} v\right\|_{L^{2}}^{2} \\
& \leq c\|v\|_{H^{q}}^{2}\|v\|_{L^{\infty}}\left\|v_{x}\right\|_{L^{\infty}}
\end{aligned}
$$

and

$$
\left|\int_{R}\left(\Lambda^{q+1} v\right) \Lambda^{q+1}\left(v^{2} v_{x}\right) d x\right| \leq c\|v\|_{H^{q+1}}^{2}\|v\|_{L^{\infty}}\left\|v_{x}\right\|_{L^{\infty}} .
$$

For the third term, using $\left\|v v_{x}\right\|_{H^{q}} \leq c\left\|\left(v^{2}\right)_{x}\right\|_{H^{q}} \leq 2 c\|v\|_{L^{\infty}}\|v\|_{H^{q+1}}$, the Cauchy-Schwarz inequality and Lemma 4.1, we obtain

$$
\begin{aligned}
\left|\int_{R}\left(\Lambda^{q} v_{x}\right) \Lambda^{q}\left(v v_{x}^{2}\right) d x\right| & \leq\left\|\Lambda^{q} v_{x}\right\|_{L^{2}}\left\|\Lambda^{q}\left(v v_{x}^{2}\right)\right\|_{L^{2}} \\
& \leq c\|v\|_{H^{q+1}}\left(\left\|v v_{x}\right\|_{L^{\infty}}\left\|v_{x}\right\|_{H^{q}}+\left\|v_{x}\right\|_{L^{\infty}}\left\|v v_{x}\right\|_{H^{q}}\right) \\
& \leq c\|v\|_{H^{q+1}}^{2}\left\|v_{x}\right\|_{L^{\infty}}\|v\|_{L^{\infty}} .
\end{aligned}
$$

For the fourth term, writing $v\left(v_{x}^{2}\right)_{x}=\left(\nu v_{x}^{2}\right)_{x}-v_{x} v_{x}^{2}$, we get

$$
\begin{aligned}
\left|\int_{R}\left(\Lambda^{q} v\right) \Lambda^{q}\left(v v_{x} v_{x x}\right) d x\right| & \leq\left|\int_{R} \Lambda^{q} v_{x} \Lambda^{q}\left(v v_{x}^{2}\right) d x\right|+\left|\int_{R} \Lambda^{q} v \Lambda^{q}\left(v_{x} v_{x}^{2}\right) d x\right| \\
& \leq c\left(\|v\|_{H^{q+1}}^{2}\left\|v_{x}\right\|_{L^{\infty}}\|v\|_{L^{\infty}}+\|v\|_{H^{q}}\left\|v_{x}^{3}\right\|_{H^{q}}\right) \\
& \leq c\|v\|_{H^{q+1}}^{2}\left(\left\|v_{x}\right\|_{L^{\infty}}\|v\|_{L^{\infty}}+\left\|v_{x}\right\|_{L^{\infty}}^{2}\right)
\end{aligned}
$$

in which we have used

$$
\begin{aligned}
\left\|v_{x}^{3}\right\|_{H^{q}} & \leq c\left(\left\|v_{x}\right\|_{L^{\infty}}\left\|v_{x}^{2}\right\|_{H^{q}}+\left\|v_{x}\right\|_{H^{q}}\left\|v_{x}^{2}\right\|_{L^{\infty}}\right) \\
& \leq c\|v\|_{H^{q+1}}\left\|v_{x}\right\|_{L^{\infty}}^{2} .
\end{aligned}
$$


Using Lemma 4.1 repeatedly results in

$$
\left|\int_{R} \Lambda^{q} v \Lambda^{q}\left(v^{2 n+1}\right) d x\right| \leq\|v\|_{H^{q}}\left\|v^{2 n+1}\right\|_{H^{q}} \leq c\|v\|_{H^{q+1}}^{2}\|v\|_{L^{\infty}}^{2 n} .
$$

For the last term in Eq. (19), using Lemma 4.1 yields

$$
\begin{aligned}
\left|\int_{R}\left(\Lambda^{q} v_{x}\right) \Lambda^{q}\left(v_{x}^{2 N-1}\right) d x\right| & \leq c\left\|v_{x}\right\|_{H^{q}}\left\|\Lambda^{q}\left(v_{x}^{2 N-1}\right)\right\|_{L^{\infty}} \\
& \leq c\|v\|_{H^{q+1}}^{2}\left\|v_{x}\right\|_{L^{\infty}}^{2 N-2} .
\end{aligned}
$$

It follows from Eqs. (20)-(25) that there exists a constant $c$ such that

$$
\begin{aligned}
& \frac{1}{2}\left|\frac{d}{d t} \int_{R}\left[\left(\Lambda^{q} v\right)^{2}+\left(\Lambda^{q} v_{x}\right)^{2}+\varepsilon\left(\Lambda^{q} v_{x x}\right)^{2}\right] d x\right| \\
& \quad \leq c\|v\|_{H^{q+1}}^{2}\left(\left\|v_{x}\right\|_{L^{\infty}}\|v\|_{L^{\infty}}+\left\|v_{x}\right\|_{L^{\infty}}^{2}+\|v\|_{L^{\infty}}^{2 n}+\left\|v_{x}\right\|_{L^{\infty}}^{2 N-2}\right) .
\end{aligned}
$$

Integrating Eq. (26) with respect to $t$ results in inequality (16).

Using the operator $\left(1-\partial_{x}^{2}\right)^{-1}$ to both sides of the first equation of system (3), we obtain

$$
\begin{aligned}
(1-\varepsilon) \nu_{t}-\varepsilon v_{t x x}= & \left(1-\partial_{x}^{2}\right)^{-1}\left[-\varepsilon v_{t}-\partial_{x}\left(\frac{4}{3} v^{3}+2 \nu v_{x}^{2}\right)\right. \\
& \left.+\frac{1}{3} \partial_{x}^{3}\left(v^{3}\right)+v v_{x} v_{x x}-\alpha v^{2 n+1}+\beta \partial_{x}\left(v_{x}^{2 N-1}\right)\right] .
\end{aligned}
$$

Applying $\left(\Lambda^{q} v_{t}\right) \Lambda^{q}$ to both sides of Eq. (27) for $q \in(0, s-1]$ gives rise to

$$
\begin{aligned}
& (1-\varepsilon) \int_{R}\left(\Lambda^{q} v_{t}\right)^{2} d x+\varepsilon \int_{R}\left(\Lambda^{q} v_{t x}\right)^{2} d x \\
& =\int_{R}\left(\Lambda^{q} v_{t}\right) \Lambda^{q-2}\left[-\varepsilon v_{t}-\partial_{x}\left(\frac{4}{3} v^{3}+2 v v_{x}^{2}+\frac{1}{3} \partial_{x}^{2} v^{3}\right)+v v_{x} v_{x x}\right. \\
& \left.\quad-\alpha v^{2 n+1}+\beta \partial_{x}\left(v_{x}^{2 N-1}\right)\right] d x
\end{aligned}
$$

In fact, we have

$$
\left|\int_{R}\left(\Lambda^{q} v_{t}\right) \Lambda^{q-2}\left(-\varepsilon v_{t}\right) d x\right| \leq \varepsilon\left\|v_{t}\right\|_{H^{q}}^{2}
$$

and

$$
\begin{aligned}
& \left|\int_{R}\left(\Lambda^{q} v_{t}\right)\left(1-\partial_{x}^{2}\right)^{-1} \Lambda^{q} \partial_{x}\left(-\frac{4}{3} v^{3}-2 v v_{x}^{2}\right) d x\right| \\
& \quad \leq c\left\|v_{t}\right\|_{H^{q}}\left(\int_{R}\left(1+\xi^{2}\right)^{q-1}\left[\int_{R}\left[-\frac{4}{3} \hat{v}^{2}(\xi-\eta) \hat{v}(\eta)-2 \widehat{v v}_{x}(\xi-\eta) \hat{v}_{x}(\eta)\right] d \eta\right]^{2}\right)^{\frac{1}{2}} \\
& \quad \leq c\left\|v_{t}\right\|_{H^{q}}\|v\|_{H^{1}}\|v\|_{H^{q+1}}\|v\|_{L^{\infty}} .
\end{aligned}
$$


We know the identity

$$
\begin{aligned}
& \int\left(\Lambda^{q} v_{t}\right)\left(1-\partial_{x}^{2}\right)^{-1} \Lambda^{q} \partial_{x}^{2}\left(v^{2} v_{x}\right) d x \\
& =-\int\left(\Lambda^{q} v_{t}\right) \Lambda^{q}\left(v^{2} v_{x}\right) d x+\int\left(\Lambda^{q} v_{t}\right)\left(1-\partial_{x}^{2}\right)^{-1} \Lambda^{q}\left(v^{2} v_{x}\right) d x .
\end{aligned}
$$

Using the Cauchy-Schwarz inequality, Lemma 4.1, $\left\|v^{2} v_{x}\right\|_{H^{q}} \leq c\|v\|_{L^{\infty}}^{2}\|v\|_{H^{q+1}}$ and $\|v\|_{L^{\infty}} \leq$ $\|v\|_{H^{1}}$ yields

$$
\begin{aligned}
\left|\int\left(\Lambda^{q} v_{t}\right) \Lambda^{q}\left(v^{2} v_{x}\right) d x\right| & \leq c\left\|v_{t}\right\|_{H^{q}}\left\|v^{2} v_{x}\right\|_{H^{q}} \\
& \leq c\left\|v_{t}\right\|_{H^{q}}\|v\|_{L^{\infty}}\|v\|_{H^{1}}\|v\|_{H^{q+1}} .
\end{aligned}
$$

Furthermore, we have

$$
\left|\int\left(\Lambda^{q} v_{t}\right)\left(1-\partial_{x}^{2}\right)^{-1} \Lambda^{q}\left(v^{2} v_{x}\right) d x\right| \leq c\left\|v_{t}\right\|_{H^{q}}\|v\|_{L^{\infty}}\|v\|_{H^{1}}\|v\|_{H^{q+1}}
$$

and

$$
\left|\int\left(\Lambda^{q} v_{t}\right)\left(1-\partial_{x}^{2}\right)^{-1} \Lambda^{q}\left(v^{2 n+1}\right) d x\right| \leq c\left\|v_{t}\right\|_{H^{q}}\|v\|_{L^{\infty}}^{2 n}\|v\|_{H^{q+1}} .
$$

For the last term in Eq. (28), we get

$$
\left|\int_{R}\left(\Lambda^{q} v_{t}\right)\left(1-\partial_{x}^{2}\right)^{-1} \Lambda^{q} \partial_{x}\left(v_{x}^{2 N-1}\right) d x\right| \leq c\left\|v_{t}\right\|_{H^{q}}\left\|v_{x}\right\|_{L^{\infty}}^{2 N-2}\|v\|_{H^{q+1}}
$$

Applying the Cauchy-Schwarz inequality and Lemmas 4.1 and 4.2 gives rise to

$$
\begin{aligned}
& \left|\int_{R}\left(\Lambda^{q} v_{t}\right)\left(1-\partial_{x}^{2}\right)^{-1} \Lambda^{q}\left(v v_{x} v_{x x}\right) d x\right| \\
& \quad \leq c\left\|v_{t}\right\|_{H^{q}}\left\|v v_{x} v_{x x}\right\|_{H^{q-2}} \\
& \quad \leq c\left\|v_{t}\right\|_{H^{q}}\left\|v\left(v_{x}^{2}\right)_{x}\right\|_{H^{q-2}} \\
& \quad \leq c\left\|v_{t}\right\|_{H^{q}}\left\|\left(v v_{x}^{2}\right)_{x}-v_{x} v_{x}^{2}\right\|_{H^{q-2}} \\
& \quad \leq c\left\|v_{t}\right\|_{H^{q}}\left(\left\|v v_{x}^{2}\right\|_{H^{q-1}}+\left\|v_{x} v_{x}^{2}\right\|_{H^{q-2}}\right) \\
& \quad \leq c\left\|v_{t}\right\|_{H^{q}}\left(\left\|v v_{x}^{2}\right\|_{H^{q}}+\left\|v_{x} v_{x}^{2}\right\|_{H^{q}}\right) \\
& \quad \leq c\left\|v_{t}\right\|_{H^{q}}\|v\|_{H^{q+1}}\left(\|v\|_{L^{\infty}}\left\|v_{x}\right\|_{L^{\infty}}+\left\|v_{x}\right\|_{L^{\infty}}^{2}\right) .
\end{aligned}
$$

Applying Eqs. (29)-(36) to Eq. (28) yields the inequality (17). The proof of Lemma 4.3 is completed.

Lemma 4.4 The following estimates hold for any $\varepsilon$ with $0<\varepsilon<\frac{1}{4}$ and $s>0$

$$
\begin{aligned}
& \left\|v_{\varepsilon 0}\right\|_{H^{q}} \leq c, \quad \text { if } q \leq s, \\
& \left\|v_{\varepsilon 0}\right\|_{H^{q}} \leq c \varepsilon^{\frac{s-q}{4}}, \quad \text { if } q>s,
\end{aligned}
$$




$$
\begin{aligned}
& \left\|v_{\varepsilon 0}-v_{0}\right\|_{H^{q}} \leq c \varepsilon^{\frac{s-q}{4}}, \quad \text { if } q \leq s, \\
& \left\|v_{\varepsilon 0}-v_{0}\right\|_{H^{s}}=o(1),
\end{aligned}
$$

where $c$ is a constant independent of $\varepsilon$.

The proof of Lemma 4.4 can be found in [13].

Remark For $s \geq 1$, using $\left\|v_{\varepsilon}\right\|_{L^{\infty}} \leq c\left\|v_{\varepsilon}\right\|_{H^{\frac{1}{2}+}} \leq c\left\|v_{\varepsilon}\right\|_{H^{1}},\left\|v_{\varepsilon}\right\|_{H^{1}}^{2} \leq c \int_{R}\left(v_{\varepsilon}^{2}+v_{\varepsilon x}^{2}\right) d x$, Eqs. (15), (37), and (38), we know

$$
\begin{aligned}
\left\|v_{\varepsilon}\right\|_{L^{\infty}}^{2} & \leq c\left\|v_{\varepsilon}\right\|_{H^{1}} \leq c \int_{R}\left(v_{\varepsilon 0}^{2}+v_{\varepsilon 0 x}^{2}+\varepsilon v_{\varepsilon 0 x x}^{2}\right) d x \\
& \leq c\left(\left\|v_{\varepsilon 0}\right\|_{H^{1}}^{2}+\varepsilon\left\|v_{\varepsilon 0}\right\|_{H^{2}}^{2}\right) \\
& \leq c\left(c+c \varepsilon \times \varepsilon^{\frac{s-2}{2}}\right) \leq c_{0},
\end{aligned}
$$

where $c_{0}$ is independent of $\varepsilon$.

Proof of Lemma 2.2 For simplicity, we use notation $v=v_{\varepsilon}$ and differentiate Eq. (27) with respect to $x$ to obtain

$$
\begin{aligned}
& (1-\varepsilon) v_{x t}-\varepsilon v_{t x x x}+\frac{1}{3} \partial_{x}^{2}\left(v^{3}\right)-\frac{3}{2} v v_{x}^{2} \\
& =v^{3}-\beta v_{x}^{2 N-1}-\Lambda^{-2}\left[\varepsilon v_{t x}+v^{3}+\frac{3}{2} v v_{x}^{2}+\frac{1}{2} \partial_{x}\left(v_{x}^{3}\right)+\alpha \partial_{x}\left(v^{2 n+1}\right)-\beta v_{x}^{2 N-1}\right] .
\end{aligned}
$$

Letting $p>0$ be an integer and multiplying the above equation by $\left(v_{x}\right)^{2 p+1}$ and then integrating the resulting equation with respect to $x$ yield

$$
\begin{aligned}
& \frac{1-\varepsilon}{2 p+2} \frac{d}{d t} \int_{R}\left(v_{x}\right)^{2 p+2} d x-\varepsilon \int_{R}\left(v_{x}\right)^{2 p+1} v_{t x x x} d x+\frac{p-1}{2 p+2} \int_{R}\left(v_{x}\right)^{2 p+3} v d x \\
& =\int_{R}\left(v_{x}\right)^{2 p+1}\left(v^{3}-\beta v_{x}^{2 N-1}\right) d x \\
& \quad-\int_{R}\left(v_{x}\right)^{2 p+1} \Lambda^{-2}\left[\varepsilon v_{t x}+v^{3}+\frac{3}{2} v v_{x}^{2}+\frac{1}{2} \partial_{x}\left(v_{x}^{3}\right)+\alpha \partial_{x}\left(v^{2 n+1}\right)-\beta v_{x}^{2 N-1}\right] d x
\end{aligned}
$$

Since

$$
-\beta \int_{R} v_{x}^{2 p+2 N} d x \leq 0,
$$

applying the Hölder inequality yields

$$
\begin{aligned}
(1-\varepsilon) & \frac{d}{d t}\left(\int_{R}\left(v_{x}\right)^{2 p+2} d x\right)^{\frac{1}{2 p+2}} \\
\leq & \varepsilon\left(\int_{R}\left|v_{t x x x}\right|^{2 p+2} d x\right)^{\frac{1}{2 p+2}}+\left(\int_{R}\left|v^{3}\right|^{2 p+2} d x\right)^{\frac{1}{2 p+2}}+\left(\int_{R}|K|^{2 p+2} d x\right)^{\frac{1}{2 p+2}} \\
& +\frac{p-1}{2 p+2}\left\|v_{x}\right\|_{L^{\infty}}\|v\|_{L^{\infty}}\left(\int_{R}\left|v_{x}\right|^{2 p+2} d x\right)^{\frac{1}{2 p+2}}
\end{aligned}
$$


where

$$
K=\Lambda^{-2}\left[\varepsilon v_{t x}+v^{3}+\frac{3}{2} v v_{x}^{2}+\frac{1}{2} \partial_{x}\left(v_{x}^{3}\right)+\alpha \partial_{x}\left(v^{2 n+1}\right)-\beta v_{x}^{2 N-1}\right] .
$$

Since $\|f\|_{L^{p}} \rightarrow\|f\|_{L^{\infty}}$ as $p \rightarrow \infty$ for any $f \in L^{\infty} \cap L^{2}$, we integrate both sides of the inequality (44) with respect to $t$ and take the limit as $p \rightarrow \infty$ to obtain

$$
\begin{aligned}
(1-\varepsilon)\left\|v_{x}\right\|_{L^{\infty}} \leq & (1-\varepsilon)\left\|v_{0 x}\right\|_{L^{\infty}} \\
& +\int_{0}^{t}\left[\varepsilon\left\|v_{t x x x}\right\|_{L^{\infty}}+c\left(\|v\|_{L^{\infty}}^{3}+\|K\|_{L^{\infty}}\right)+\frac{1}{2}\|v\|_{L^{\infty}}\left\|v_{x}\right\|_{L^{\infty}}^{2}\right] d \tau .
\end{aligned}
$$

Using the inequality (41) yields

$$
\left\|\Lambda^{-2} v^{3}\right\|_{L^{\infty}} \leq c, \quad\left\|\Lambda^{-2} \partial_{x}\left(v^{2 n+1}\right)\right\|_{L^{\infty}} \leq c .
$$

Using Eq. (46), Lemma 4.1, and Lemma 4.3 gives rise to

$$
\begin{aligned}
\|K\|_{L^{\infty}} & \leq c\left\|\Lambda^{-2}\left[\varepsilon v_{t x}+\frac{3}{2} v v_{x}^{2}+\frac{1}{2} \partial_{x}\left(v_{x}^{3}\right)-\beta v_{x}^{2 N-1}\right]\right\|_{H^{\frac{1}{2}+}}+c \\
& \leq c\left(\left\|\Lambda^{-2} v_{t x}\right\|_{H^{\frac{1}{2}+}}+\left\|\Lambda^{-2}\left(v v_{x}^{2}\right)\right\|_{H^{\frac{1}{2}+}}+\left\|\Lambda^{-2} \partial_{x}\left(v_{x}^{3}\right)\right\|_{H^{\frac{1}{2}+}}+\left\|v_{x}^{2 N-1}\right\|_{H^{0}}\right)+c \\
& \leq c\left(\left\|v_{t}\right\|_{L^{2}}+\left\|v v_{x}^{2}\right\|_{H^{0}}+\left\|v_{x}^{3}\right\|_{H^{0}}+\left\|v_{x}^{2 N-1}\right\|_{H^{0}}\right)+c \\
& \leq c\left(\left\|v_{t}\right\|_{L^{2}}+\left\|v_{x}\right\|_{L^{\infty}}+\left\|v_{x}\right\|_{L^{\infty}}^{2}+\left\|v_{x}\right\|_{L^{\infty}}^{2 N-2}\right)+c,
\end{aligned}
$$

where $c$ is a constant independent of $\varepsilon$. Using Eqs. (17) and (47), we have

$$
\int_{0}^{t}\|K\|_{L^{\infty}} d \tau \leq c \int_{0}^{t}\left(1+\left\|v_{x}\right\|_{L^{\infty}}+\left\|v_{x}\right\|_{L^{\infty}}^{2}+\left\|v_{x}\right\|_{L^{\infty}}^{2 N-2}\right) d \tau
$$

where $c$ is a constant independent of $\varepsilon$. Moreover, for any fixed $r \in\left(\frac{1}{2}, 1\right)$, there exists a constant $c_{r}$ such that $\left\|v_{x x x t}\right\|_{L^{\infty}} \leq c_{r}\left\|v_{x x x t}\right\|_{H^{r}} \leq c_{r}\left\|v_{t}\right\|_{H^{r+3}}$. Using Eqs. (17) and (41) yields

$$
\left\|v_{t x x x}\right\|_{L^{\infty}} \leq c\|v\|_{H^{r+4}}\left(1+\left\|v_{x}\right\|_{L^{\infty}}+\left\|v_{x}\right\|_{L^{\infty}}^{2}+\left\|v_{x}\right\|_{L^{\infty}}^{2 N-2}\right) .
$$

Making use of the Gronwall inequality in Eq. (16) with $q=r+3, v=v_{\varepsilon}$ and Eq. (41) gives rise to

$$
\begin{aligned}
\|v\|_{H^{r+4}}^{2} \leq & {\left[\left(\Lambda^{r+4} u_{0}\right)^{2}+\varepsilon\left(\Lambda^{r+3} v_{0 x x}\right)^{2}\right] } \\
& \times \exp \left[c \int_{0}^{t}\left(1+\left\|v_{x}\right\|_{L^{\infty}}+\left\|v_{x}\right\|_{L^{\infty}}^{2}+\left\|v_{x}\right\|_{L^{\infty}}^{2 N-2}\right) d \tau\right] .
\end{aligned}
$$

From Eqs. (37), (38), (49), and (50), one has

$$
\begin{aligned}
\left\|v_{t x x x}\right\|_{L^{\infty}} \leq & c \varepsilon^{\frac{s-r-4}{4}}\left(1+\left\|v_{x}\right\|_{L^{\infty}}+\left\|v_{x}\right\|_{L^{\infty}}^{2}+\left\|v_{x}\right\|_{L^{\infty}}^{2 N-2}\right) \\
& \times \exp \left[c \int_{0}^{t}\left(1+\left\|v_{x}\right\|_{L^{\infty}}+\left\|v_{x}\right\|_{L^{\infty}}^{2}+\left\|v_{x}\right\|_{L^{\infty}}^{2 N-2}\right) d \tau\right] .
\end{aligned}
$$


For $\varepsilon<\frac{1}{4}$ and $s>r$, it follows from Eqs. (45), (48), and (51) that

$$
\begin{aligned}
\left\|v_{x}\right\|_{L^{\infty}} \leq & \left\|u_{0 x}\right\|_{L^{\infty}}+c \int_{0}^{t}\left[\varepsilon^{\frac{s-r}{4}}\left(1+\left\|v_{x}\right\|_{L^{\infty}}+\left\|v_{x}\right\|_{L^{\infty}}^{2}+\left\|v_{x}\right\|_{L^{\infty}}^{2 N-2}\right)\right. \\
& \times \exp \left(c \int_{0}^{\tau}\left(1+\left\|v_{x}\right\|_{L^{\infty}}+\left\|v_{x}\right\|_{L^{\infty}}^{2}+\left\|v_{x}\right\|_{L^{\infty}}^{2 N-2}\right) d \varsigma\right) \\
& \left.+1+\left\|v_{x}\right\|_{L^{\infty}}+\left\|v_{x}\right\|_{L^{\infty}}^{2}+\left\|v_{x}\right\|_{L^{\infty}}^{2 N-2}\right] d \tau .
\end{aligned}
$$

It follows from the contraction-mapping principle that there is a $T>0$ such that the equation

$$
\begin{aligned}
\|W\|_{L^{\infty}}= & \left\|u_{0 x}\right\|_{L^{\infty}}+c \int_{0}^{t}\left[\left(1+\|W\|_{L^{\infty}}+\|W\|_{L^{\infty}}^{2}+\|W\|_{L^{\infty}}^{2 N-2}\right)\right. \\
& \times \exp \left(c \int_{0}^{\tau}\left(1+\|W\|_{L^{\infty}}+\|W\|_{L^{\infty}}^{2}+\|W\|_{L^{\infty}}^{2 N-2}\right) d \varsigma\right) \\
& \left.+1+\|W\|_{L^{\infty}}+\|W\|_{L^{\infty}}^{2}+\|W\|_{L^{\infty}}^{2 N-2}\right] d \tau
\end{aligned}
$$

has a unique solution $W \in C[0, T]$. Using the theorem at p.51 in [12] shows that there are constants $T>0$ and $c>0$ independent of $\varepsilon$ such that $\left\|u_{x}\right\|_{L^{\infty}} \leq W(t)$ for arbitrary $t \in[0, T]$, which leads to the conclusion of Lemma 2.2 .

Using the conclusion of Lemmas 2.2 and 4.3, Eq. (41), $q \in[0, s-1]$, the notation $v_{\varepsilon}=v$, and the Gronwall inequality results in the inequalities

$$
\left\|v_{\varepsilon}\right\|_{H^{q}} \leq\left\|v_{\varepsilon}\right\|_{H^{q+1}} \leq c \exp \left[c \int_{0}^{t}\left(1+\left\|v_{x}\right\|_{L^{\infty}}+\left\|v_{x}\right\|_{L^{\infty}}^{2}+\left\|v_{x}\right\|_{L^{\infty}}^{2 N-2}\right) d \tau\right] \leq c
$$

and

$$
\left\|v_{\varepsilon t}\right\|_{H^{r}} \leq c\left(1+\left\|v_{x}\right\|_{L^{\infty}}+\left\|v_{x}\right\|_{L^{\infty}}^{2}+\left\|v_{x}\right\|_{L^{\infty}}^{2 N-2}\right)\left\|v_{\varepsilon}\right\|_{H^{r+1}} \leq c,
$$

where $r \in(0, s-1]$ and any $t \in[0, T)$. It follows from Aubin's compactness theorem that there is a subsequence of $\left\{v_{\varepsilon}\right\}$, denoted by $\left\{v_{\varepsilon_{n}}\right\}$, such that $\left\{v_{\varepsilon_{n}}\right\}$ and their temporal derivatives $\left\{v_{\varepsilon_{n}}\right\}$ are weakly convergent to a function $v(t, x)$ and its derivative $v_{t}$ in $L^{2}\left([0, T], H^{s}(R)\right)$ and $L^{2}\left([0, T], H^{s-1}(R)\right)$, respectively. Moreover, for any real number $R_{1}>0,\left\{v_{\varepsilon_{n}}\right\}$ is convergent to the function $v$ strongly in the space $L^{2}\left([0, T], H^{q_{1}}\left(-R_{1}, R_{1}\right)\right)$ for $q_{1} \in[0, s)$ and $\left\{v_{\varepsilon_{n}}\right\}$ converges to $v_{t}$ strongly in the space $L^{2}\left([0, T], H^{r}\left(-R_{1}, R_{1}\right)\right)$ for $r \in[0, s-1]$. Now, we can prove the existence of a weak solution to Eq. (2).

Proof of Theorem 2.1 From Lemma 2.2, we know that $\left\{v_{\varepsilon_{n} x}\right\}\left(\varepsilon_{n} \rightarrow 0\right)$ is bounded in the space $L^{\infty}$. Thus, the sequences $\left\{v_{\varepsilon_{n}}\right\}$ and $\left\{v_{\varepsilon_{n} x}^{j}\right\}(j=1,2,2 N-2)$ are weakly convergent to $v$ and $v_{x}^{j}$ in $L^{2}\left([0, T], H^{r}(-R, R)\right)$ for any $r \in[0, s-1)$, respectively. Therefore, $v$ satisfies the equation

$$
\begin{aligned}
-\int_{0}^{T} \int_{R} v\left(g_{t}-g_{t x x}\right) d x d t= & \int_{0}^{T} \int_{R}\left[\left(\frac{4}{3} v^{3}+2 v v_{x}^{2}\right) g_{x}-\frac{1}{3} v^{3} g_{x x x}\right. \\
& \left.-\frac{1}{2} v_{x}^{3} g-\frac{1}{2} v_{x}^{2} v g_{x}+\alpha v^{2 n+1} g-\beta v_{x}^{2 N-1} g_{x}\right] d x d t
\end{aligned}
$$


with $v(0, x)=v_{0}(x)$ and $g \in C_{0}^{\infty}$. Since $X=L^{1}([0, T] \times R)$ is a separable Banach space and $\left\{v_{\varepsilon_{n} x}\right\}$ is a bounded sequence in the dual space $X^{*}=L^{\infty}([0, T] \times R)$ of $X$, there exists a subsequence of $\left\{v_{\varepsilon_{n} x}\right\}$, still denoted by $\left\{v_{\varepsilon_{n} x}\right\}$, weakly star convergent to a function $u$ in $L^{\infty}([0, T] \times R)$. One derives from the $\left\{v_{\varepsilon_{n} x}\right\}$ being weakly convergent to $v_{x}$ in $L^{2}([0, T] \times R)$ that $v_{x}=u$ almost everywhere. Thus, we obtain $v_{x} \in L^{\infty}([0, T] \times R)$.

Competing interests

The author declares that he has no competing interests.

\section{Author's contributions}

The article is work of one author, who approves the final manuscript.

\section{Acknowledgements}

This work is supported by the Fundamental Research Funds for the Central Universities (JBK120504).

Received: 4 February 2014 Accepted: 27 May 2014 Published: 04 Jun 2014

\section{References}

1. Novikov, V: Generalizations of the Camassa-Holm equation. J. Phys. A, Math. Theor. 42, 342002 (2009)

2. Hone, ANW, Wang, JP: Integrable peakon equations with cubic nonlinearity. J. Phys. A, Math. Theor. 41, 372002 (2008)

3. Himonas, A, Holliman, C: The Cauchy problem for the Novikov equation. Nonlinearity 25, 449-479 (2012)

4. Himonas, A, Holmes, J: Hölder continuity of the solution map for the Novikov equation. J. Math. Phys. 54, 061501 (2013)

5. Ni, L, Zhou, Y: Well-posedness and persistence properties for the Novikov equation. J. Differ. Equ. 250, 3002-3021 (2011)

6. Tiglay, F: The periodic Cauchy problem for Novikov equation. Int. Math. Res. Not. 20, 4633-4648 (2011)

7. Hone, AN, Lundmark, H, Szmigielski, J: Explicit multipeakon solutions of Novikov's cubically nonlinear integrable Camassa-Holm type equation. Dyn. Partial Differ. Equ. 6, 253-289 (2009)

8. Camassa, R, Holm, D: An integrable shallow water equation with peaked solitons. Phys. Rev. Lett. 71, 1661-1664 (1993)

9. Constantin, A, Lannes, D: The hydro-dynamical relevance of the Camassa-Holm and Degasperis-Procesi equations. Arch. Ration. Mech. Anal. 193, 165-186 (2009)

10. Constantin, A, Escher, J: Wave breaking for nonlinear nonlocal shallow water equations. Acta Math. 181, 229-243 (1998)

11. Rodriguez-Blanco, G: On the Cauchy problem for the Camassa-Holm equation. Nonlinear Anal. TMA 46, 309-327 (2001)

12. Li, Y, Olver, P: Well-posedness and blow-up solutions for an integrable nonlinearly dispersive model wave equation. J. Differ. Equ. 162, 27-63 (2000)

13. Lai, SY, Wu, YH: The local well-posedness and existence of weak solutions for a generalized Camassa-Holm equation. J. Differ. Equ. 248, 2038-2063 (2010)

14. Kato, T, Ponce, G: Commutator estimates and the Euler and Navier-Stokes equations. Commun. Pure Appl. Math. 41 891-907 (1988)

10.1186/1029-242X-2014-230

Cite this article as: Lai: The investigation of local weak solutions for a generalized Novikov equation. Journal of Inequalities and Applications 2014, 2014:230

\section{Submit your manuscript to a SpringerOpen ${ }^{\ominus}$ journal and benefit from:}

- Convenient online submission

Rigorous peer review

- Immediate publication on acceptance

- Open access: articles freely available online

- High visibility within the field

- Retaining the copyright to your article 